\title{
Generalized erythematous and scaly plaques and papules: a rare case of Rosai-Dorfman disease accompanied by multiple myeloma*
}

\author{
Anoosh Shafiee ${ }^{1}$
}

\author{
Soheila Nasiri ${ }^{1}$
}

DOI: http:/ / dx.doi.org/10.1590/abd1806-4841.20187269

\begin{abstract}
A 75-year-old male presented with generalized erythematous, scaly plaques and painless lymphadenopathy. Rosai-Dorfman disease was suspected based on clinical manifestations and confirmed by histopathologic and immune reactivity studies performed on the biopsy obtained from the left supraclavicular lymph node. The patient was also diagnosed with multiple myeloma according to urine electrophoresis, serum light chain assay, and bone marrow biopsy, which were initially performed for evaluation of anemia. This report highlights the dermatological manifestations of Rosai-Dorfman disease with generalized painless lymphadenopathy.
\end{abstract}

Keywords: Case reports; Histiocytosis, sinus; Multiple myeloma

\section{INTRODUCTION}

Rosai-Dorfman disease (RDD) or sinus histiocytosis was first described in 1969 by Rosai and Dorfman as a rare entity characterized by non-neoplastic proliferation of macrophage-histiocyte cells with massive and generalized lymphadenopathies, most commonly affecting healthy individuals with no underlying diseases. ${ }^{1,2}$ Massive enlarged lymph nodes with fever, elevated erythrocyte sedimentation rate (ESR), and polyclonal hypergammaglobulinemia can be the first manifestations of RDD. ${ }^{3}$

Review of the available literature yields scant reports of RDD cases with cutaneous involvement, none of which were associated with multiple myeloma. We present a case of RDD with generalized erythematous scaly plaques and painless lymphadenopathies that met the diagnostic criteria for multiple myeloma as well.

\section{CASE REPORT}

A 75-year-old male presented to our dermatology clinic with persistent and progressive erythematous, scaly plaques and papules that had initially appeared two years previously. The first cutaneous lesion developed on the abdominal skin and gradually spread to the entire surface of the body. During this period some of the lesions regressed spontaneously, leaving scars (Figure 1). The patient had no constitutional symptoms (i.e., fever, weight loss, night sweats, or malaise), and his past medical and surgical history was unremarkable. He denied taking any medications.

On admission, vital signs were stable and a comprehensive physical examination revealed erythematous scaly plaques and papules over the entire surface of the body, nodular lesions in the lower extremities along with non-tender, firm, and mobile palpable lymph nodes in the left axillary, left supraclavicular, and bilateral inguinal regions, whose maximum diameters were measured by $\mathrm{CT}$ scan to be $3 \mathrm{~cm}, 0.8 \mathrm{~cm}$, and $1.7 \mathrm{~cm}$, respectively.

On laboratory evaluation, the results of complete blood count $(\mathrm{CBC})$ and differentials were within normal limits except for a low hemoglobin level of $10 \mathrm{~g} / \mathrm{dl}$. Reticulocyte count was $2 \%$. Biochemistry panel, viral assays, collagen vascular markers, and lactate dehydrogenase level were also in the normal range. Erythrocyte sedimentation rate and $\mathrm{C}$-reactive protein were high $(\mathrm{ESR}=$ $90 \mathrm{~mm} / \mathrm{h}, \mathrm{CRP}=62 \mathrm{mg} / \mathrm{l}$ ). CA19-9 and CA15-3 levels were $3.5 \mathrm{U} / \mathrm{ml}$ and $17.9 \mathrm{U} / \mathrm{ml}$, respectively (within the normal range).

The patient underwent abdominal and pelvic ultrasound assessment which only revealed the two non-reactive enlarged lymph nodes in the left axillary region with a maximum short axis

\footnotetext{
Received 03 May 2017.

Accepted 18 July 2017.

* Work conducted at the Dermatology Clinic of Loghman-e-Hakim Hospital, Shahid Beheshti University of Medical Sciences, Tehran, Iran.

Financial support: None.

Conflict of interest: None.

1 Skin Research Center, Shahid Beheshti University of Medical Sciences, Tehran, Iran.
}

MAILING ADDRESS:

Soheila Nasiri

E-mail: dr.nasiri.soheila@gmail.com

C2018 by Anais Brasileiros de Dermatologia 
of $30 \mathrm{~mm}$ and another non-reactive lymph node in the supraclavicular region measuring $8 \mathrm{~mm}$ on its short axis.

Histopathological evaluation of skin tissue biopsy revealed an unremarkable epidermis, but the underlying dermis was found to be ill-defined, with moderately dense infiltration of foamy histiocytes with abundant pale or xanthomatous cytoplasm admixed with a few touton giant cells, some lymphocytes, many plasma cells, neutrophils, and rare eosinophils around the blood vessels or dispersed within the dermis showing scattered lymphophagocytosis in histiocytic cells. Axillary lymph node biopsy presented with diffusely distributed histiocytes throughout the smears, with multiple lobules or nuclei. No nuclear atypia, evidence of malignancy, or nuclear grooving was noted. Histiocytes had fine chromatin with inconspicuous prominent nucleoli and abundant pale cytoplasm and contained numerous intact lymphocytes and plasma cells, a characteristic finding for Rosai-Dorfman disease known as emperipolesis. In some of these histiocytes, the great number of phagocytosed cells caused obscuration of the nucleus (Figure 2). Immunohistochemistry (IHC) staining was also carried out, and the histiocytic cells were found to be positive for S100 and CD68 proteins but negative for CD1a (Figure 3). The mixed lymphohistiocytic inflammatory cell infiltrate in the dermis along with the lymphophagocytosis (emperipolesis) and IHC staining patterns were consistent with the clinically suspected diagnosis of Rosai-Dorfman disease.

Moreover, the left supraclavicular lymphadenopathy and anemia raised suspicion of gastrointestinal cancer, so the patient underwent an upper gastrointestinal endoscopy, which was normal. All of the tumor markers were also negative, so malignancy was ruled out.

The results of urine protein electrophoresis were suggestive of multiple myeloma with light chain $=8.3 \mathrm{mg} / \mathrm{dl}$, apoprotein $\mathrm{A} 1=1.8 \mathrm{mg} / \mathrm{dl}$, alpha1-microglobulin= $4.4 \mathrm{mg} / \mathrm{dl}$, albumin=3mg/ $\mathrm{dl}$, and $\mathrm{IgG} / \mathrm{IgA}=0.4 \mathrm{mg} / \mathrm{dl}$. C3, C4, and CH50 levels were $129.4 \mathrm{mg} / \mathrm{dl}$, $20.8 \mathrm{mg} / \mathrm{dl}$, and $94 \%$, respectively.

Further assessments for multiple myeloma included serum free light chain assay by fixed-time nephelometry, and bone marrow biopsy. Serum free kappa was $0.16 \mathrm{mg} / \mathrm{dl}$, serum free lambda was $6.7 \mathrm{mg} / \mathrm{dl}$, and the kappa-to-lambda ratio was 0.024 . Bone marrow biopsy revealed more than $15 \%$ plasma cells, also indicative of multiple myeloma.

Accordingly, based on the medical history, clinical findings, laboratory tests, histopathology, and bone marrow biopsy, the pa-
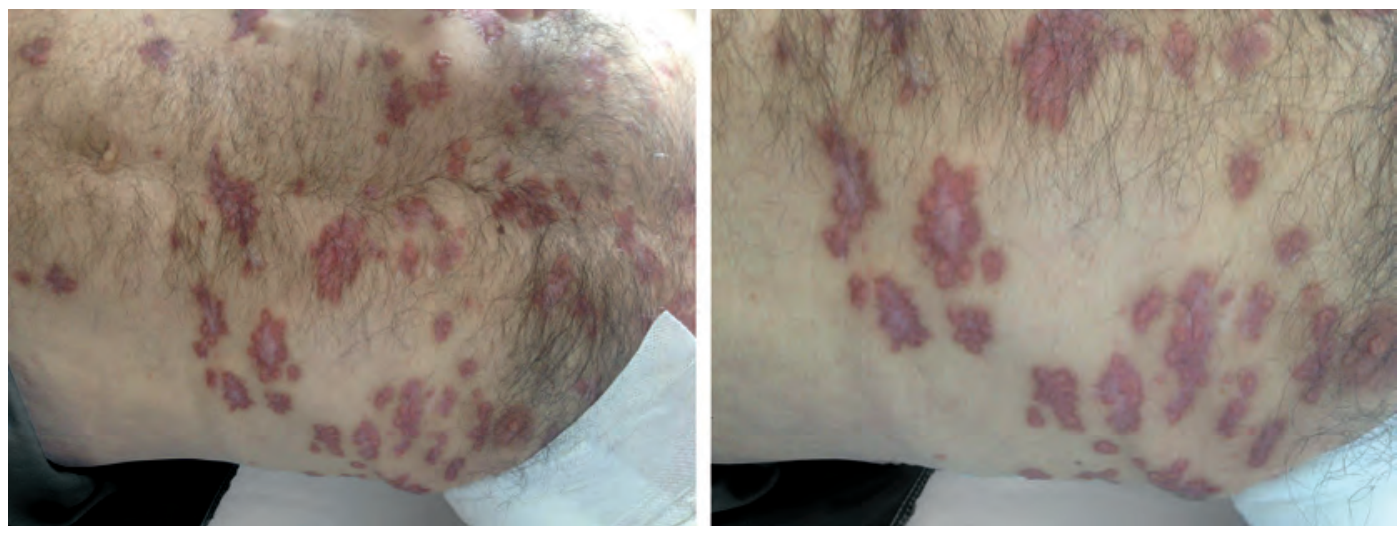

Figure 1: Erythematous and scaly plaques and papules over the entire surface of the body
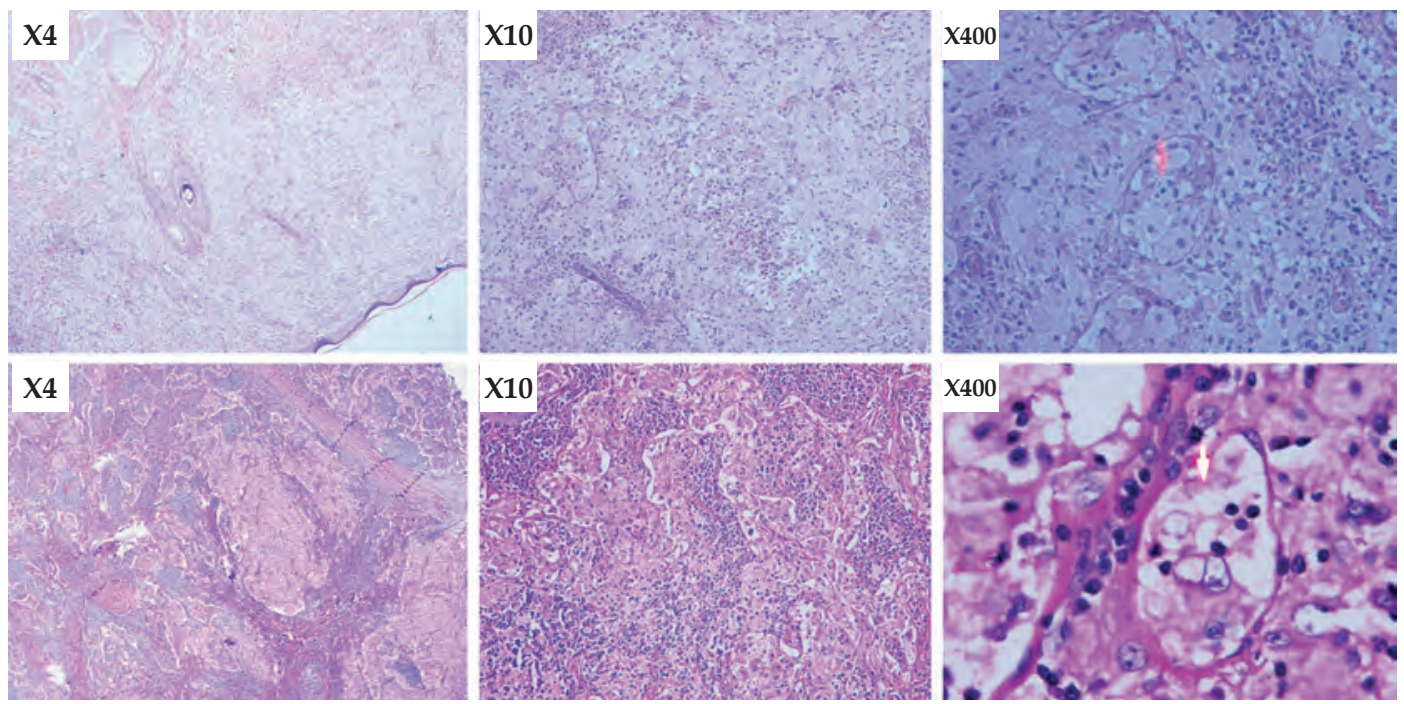

Figure 2:

histopathological findings of skin tissue biopsy (top row) and axillary lymph node biopsy (arrow shows emperipolesis) Hematoxylin \& eosin, X10-X400 

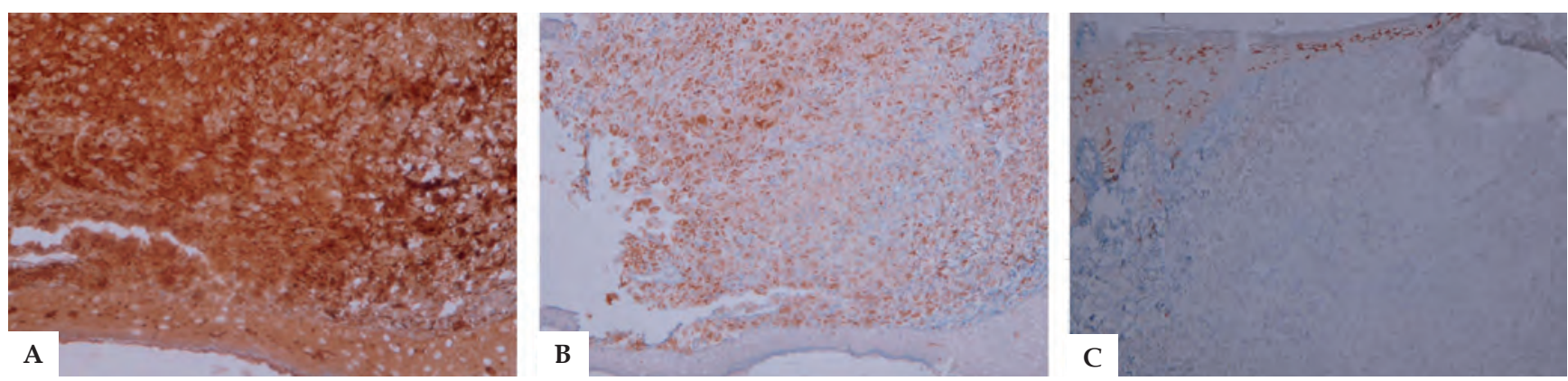

Figure 3: Immunohistochemistry, X10; findings of A - S100, B - CD68, and C - CD1a proteins

tient was referred to an oncologist with simultaneous diagnoses of multiple myeloma and RDD for further assessment and treatment.

\section{DISCUSSION}

Most patients with Rosai-Dorfman disease present with generalized lymphadenopathy as the first manifestation,,$^{2,4}$ and thirty to forty percent of patients present with involvement of axillary, mediastinal, or inguinal lymph nodes. ${ }^{2}$ In our patient the supraclavicular, inguinal, and axillary lymph nodes were involved. Eighty percent of reported RDD cases are under 20 years of age, and this disorder in our patient who was in his seventies is quite rare. ${ }^{5}$

Unlike most RDD cases that present with night sweats, malaise, and weight loss, our patient reported no constitutional symptoms. On the other hand, histopathological evaluation of his skin biopsy revealed the characteristic finding of RDD known as emperipolesis or lymphophagocytosis, defined as presence of intact lymphocytes within the cytoplasm of large histiocytes. ${ }^{4}$

Although RDD has been reported in association with various diseases such as clear cell sarcoma, IgG4-related disease, or masquerading as cholangiocarcinoma, to date no reports have been published of a patient meeting the diagnostic criteria of both multiple myeloma and RDD. ${ }^{6-8}$ The unique characteristics of the patient described in this article that made him a rare case worth reporting were his age, which was did not fall in the typical age bracket of patients with RDD, his chief complaint of skin lesions which is a rare manifestation of the disease, and his laboratory results which were found to be consistent with the diagnostic criteria for multiple myeloma.

\section{REFERENCES}

1. Rosai J, Dorfman RF. Sinus histiocytosis with massive lymphadenopathy: a newly recognized benign clinicopathological entity. Arch Pathol. 1969;87:63-70.

2. Kushwaha R, Ahluwalia C, Sipayya V. Diagnosis of sinus histiocytosis with massive lymphadenopathy (Rosai-Dorfman Disease) by fine needle aspiration cytology. J Cytol. 2009;26:83-5.

3. Gupta P, Babyn P. Sinus histiocytosis with massive lymphadenopathy (RosaiDorfman disease): a clinicoradiological profile of three cases including two with skeletal disease. Pediatr Radiol. 2008:38:721-8.

4. Sharma S, Bhardwaj S, Hans D. Rosai-Dorfman Disease. JK science. 2010;12:194-6.

5. Al-Khateeb TH. Cutaneous Rosai-Dorfman Disease of the Face: A Comprehensive Literature Review and Case Report. J Oral Maxillofac Surg. 2016;74:528-40.
6. Hassani J, Porubsky C, Berman C, Zager J, Messina J, Henderson-Jackson E. Intraperitoneal Rosai-Dorfman disease associated with clear cell sarcoma: first case report. Pathology. 2016;48:742-744.

7. Menon MP, Evbuomwan MO, Rosai J, Jaffe ES, Pittaluga S. A subset of RosaiDorfman disease cases show increased IgG4-positive plasma cells: another red herring or a true association with IgG4-related disease? Histopathology. 2014;64:455-9.

8. Teymoorian A, Earl TM, Borg BB. Rosai-Dorfman Disease Masquerading as Cholangiocarcinoma. Clin Gastroenterol Hepatol. 2017;15:e55-e56.

$\begin{array}{ll}\text { Anoosh Shafiee } & \text { (D) ORCID } 0000-0001-8282-6241 \\ \text { Soheila Nasiri } & \text { (iD) ORCID } 0000-0002-1131-1870\end{array}$

How to cite this article: Shafiee A, Nasiri S. Generalized erythematous and scaly plaques and papules: A rare case of Rosai Dorfman accompanied by multiple myeloma. An Bras Dermatol. 2018;93(3):432-4. 